\title{
Antigen-triggered logic-gating of DNA nanodevices
}

Wouter Engelen ${ }^{1,2}$, Christian Sigl ${ }^{1,2}$, Karoline Kadletz $^{1,2}$, Elena M. Willner ${ }^{1,2}$ and Hendrik Dietz ${ }^{1,2, *}$

${ }^{1}$ Laboratory for Biomolecular Nanotechnology, Physics Department, Technical University of Munich, Garching near Munich, 85748, Germany

${ }^{2}$ Munich Institute of Biomedical Engineering, Technical University of Munich, Garching near Munich, 85748, Germany

Please address correspondence to: $\underline{\operatorname{dietz} @ \text { tum.de }}$

Content:

Supplementary Figure 1-5

Supplementary Note 1-2 


\section{Supplementary Figures}
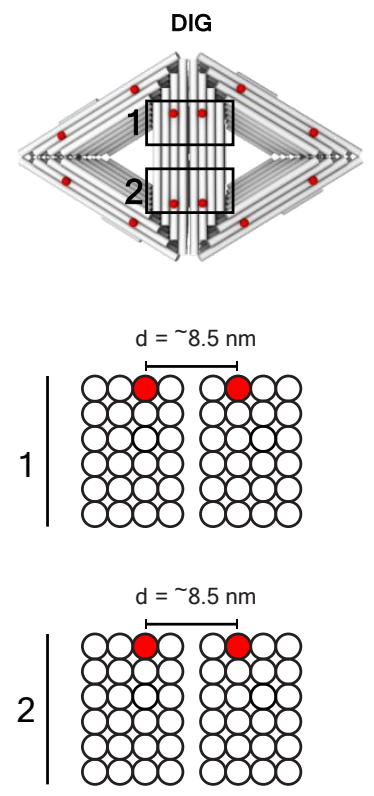
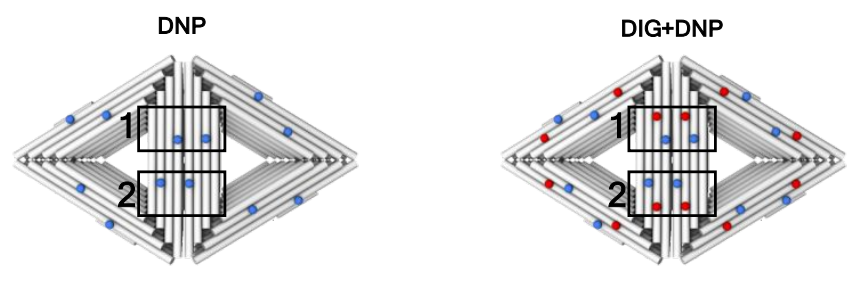

1
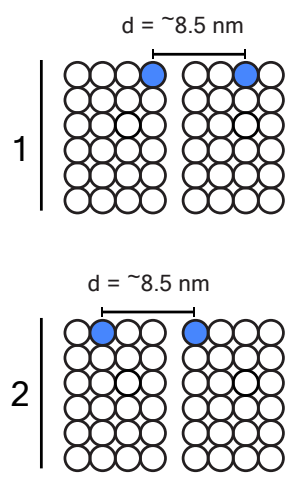
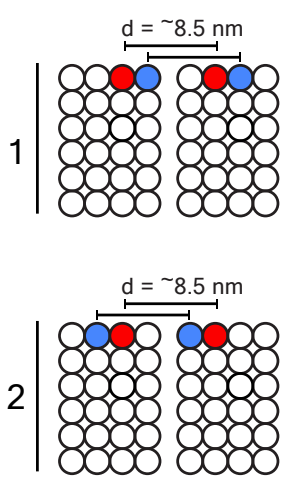

Supplementary Figure 1 | Schematic representation of digoxigenin and/or 2,4-dinitrophenol antigen placement on triangle subunits. For clarity a triangle dimer is shown with colored spheres representing individual antigens. Upon formation of a triangle-triangle interface the antigens located on opposing triangles form a single antibody docking site with antigen spacing of $\sim 8.5$ $\mathrm{nm}$, yielding 2 antibody docking sites per triangle-triangle interface for each antibody-antigen pair.

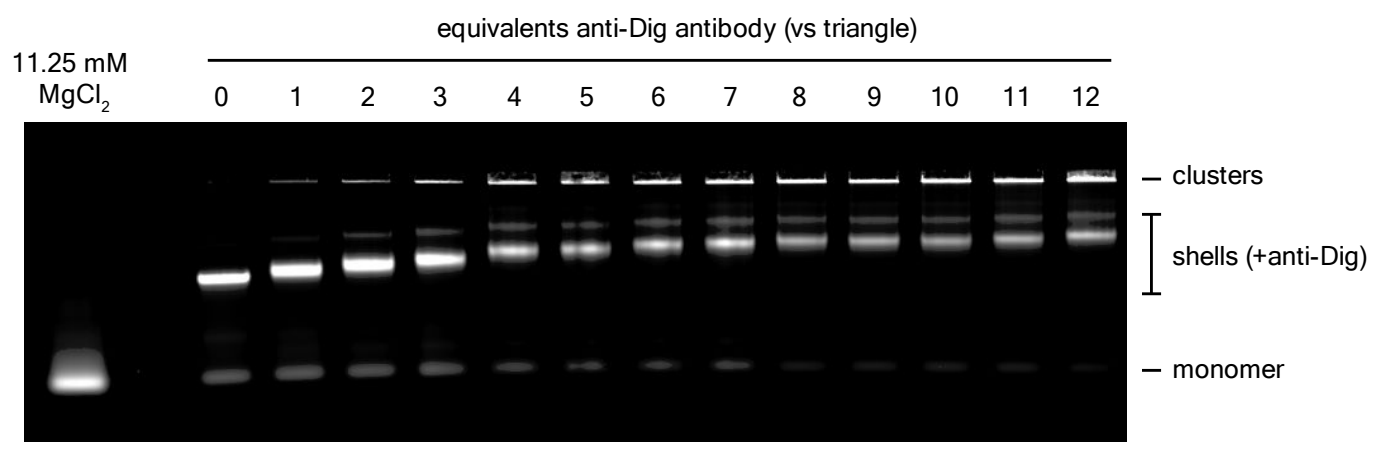


Supplementary Figure 2 | Titration of anti-Dig antibody to assembled shells. $0.75 \%$ agarose gel containing 0.5xTBE buffer and $22.5 \mathrm{mM} \mathrm{MgCl} 2$ showing a titration of increasing equivalents of anti-Dig antibody to assembled shells. Increasing amounts of anti-Dig antibody were titrated to 1 $\mathrm{nM}$ assembled shells at $25 \mathrm{mM} \mathrm{MgCl}$. A control sample was included of shells diluted to 11.25 $\mathrm{MgCl}_{2}$, revealing a clear triangle monomer band.
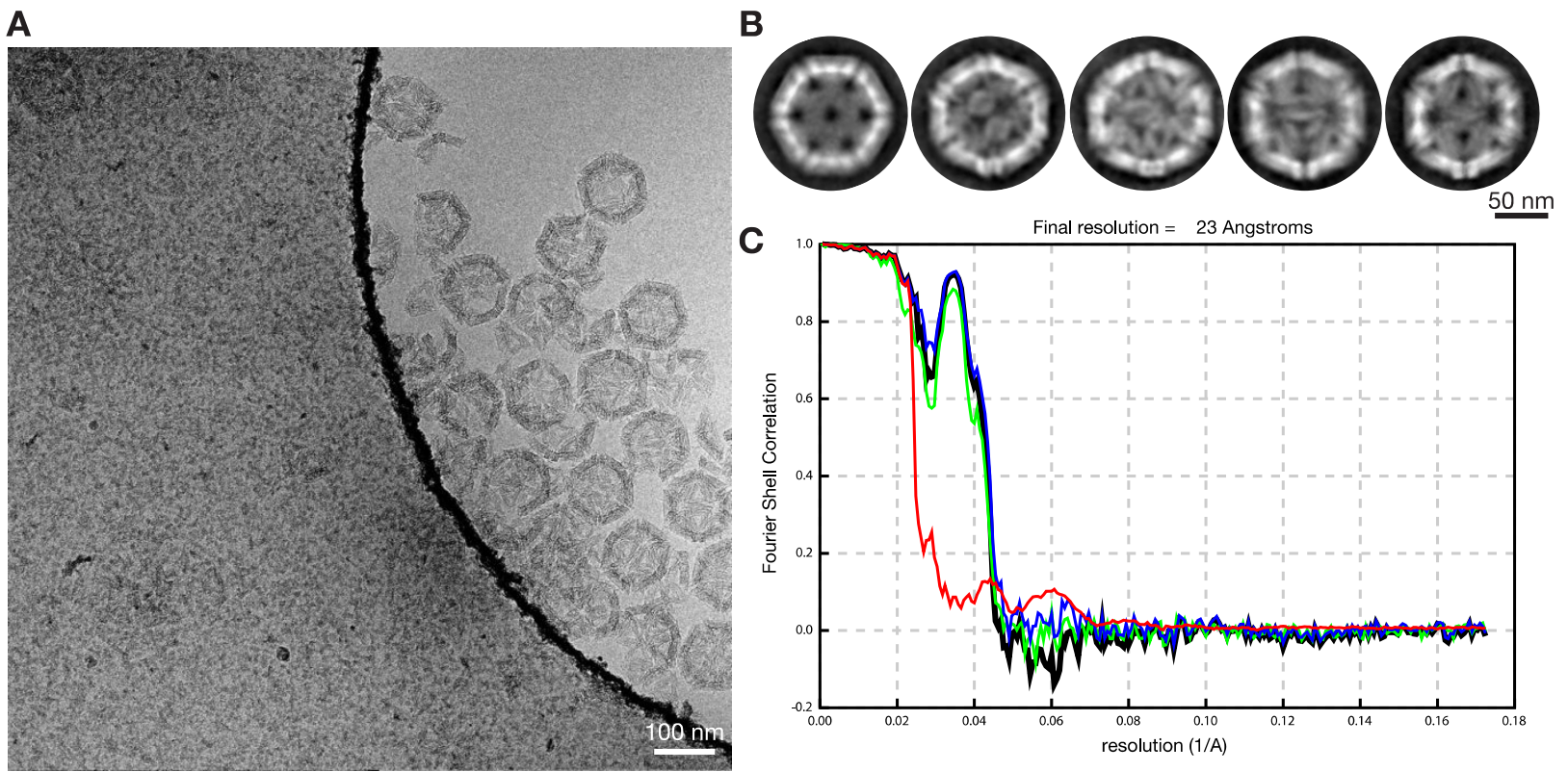

C

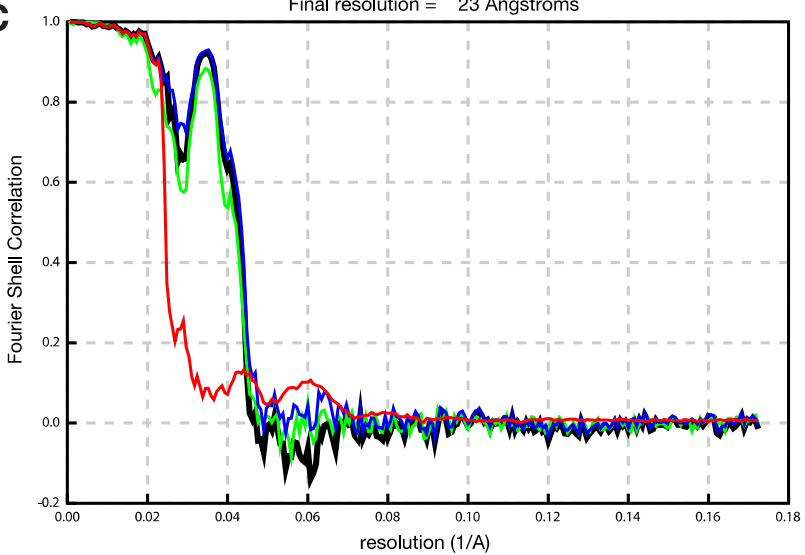

D
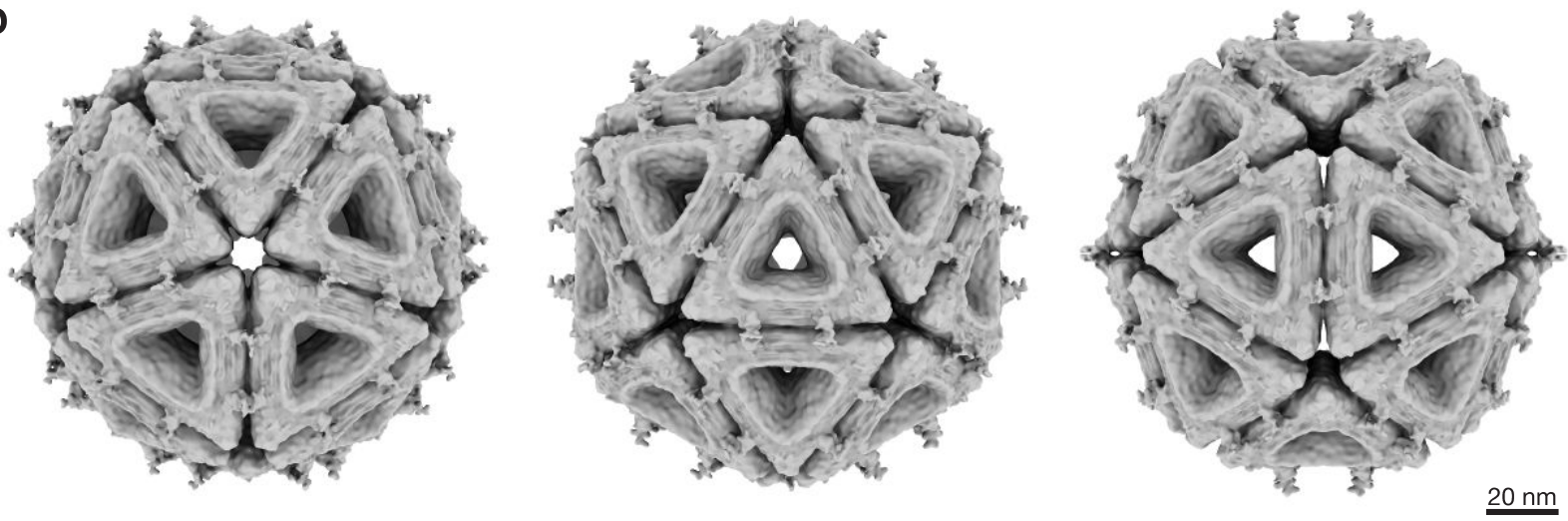

Supplementary Figure 3 | Cryo-EM single particle reconstruction of $T=1$ shells stabilized with antiDig antibodies at $25 \mathrm{mM} \mathrm{MgCl}_{2}$. (A) Cryo-EM micrograph of $\mathrm{T}=1$ shells shell stabilized by 60 antiDig antibodies in free-standing ice. (B) Two-dimensional class averages showing different orientations. (C) Graph showing different FSC curves which were used for resolution estimation. (D) Electron density map shown from the 5-fold, 3-fold and 2-fold symmetry axis (left to right). 

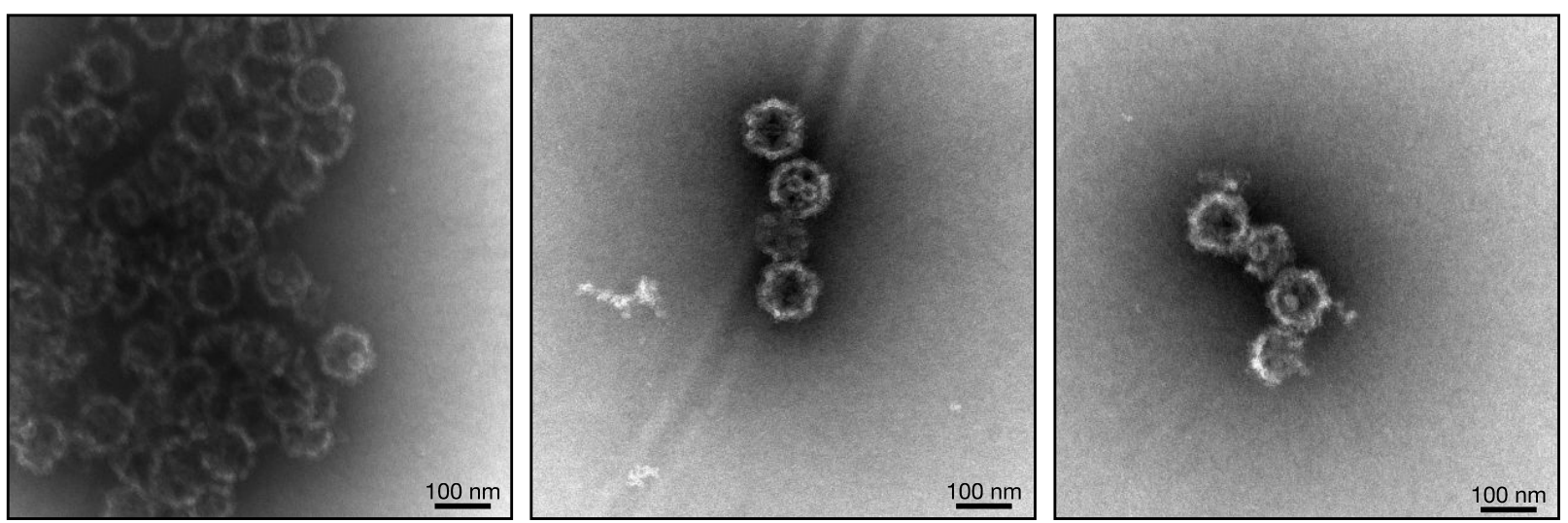

Supplementary Figure 4 | Additional negative stained TEM images of HBVc particles encapsulated by spring loaded $\mathrm{T}=1$ shells at $12 \mathrm{mM} \mathrm{MgCl}$. Additional images for the sample shown in Fig. 4c. 


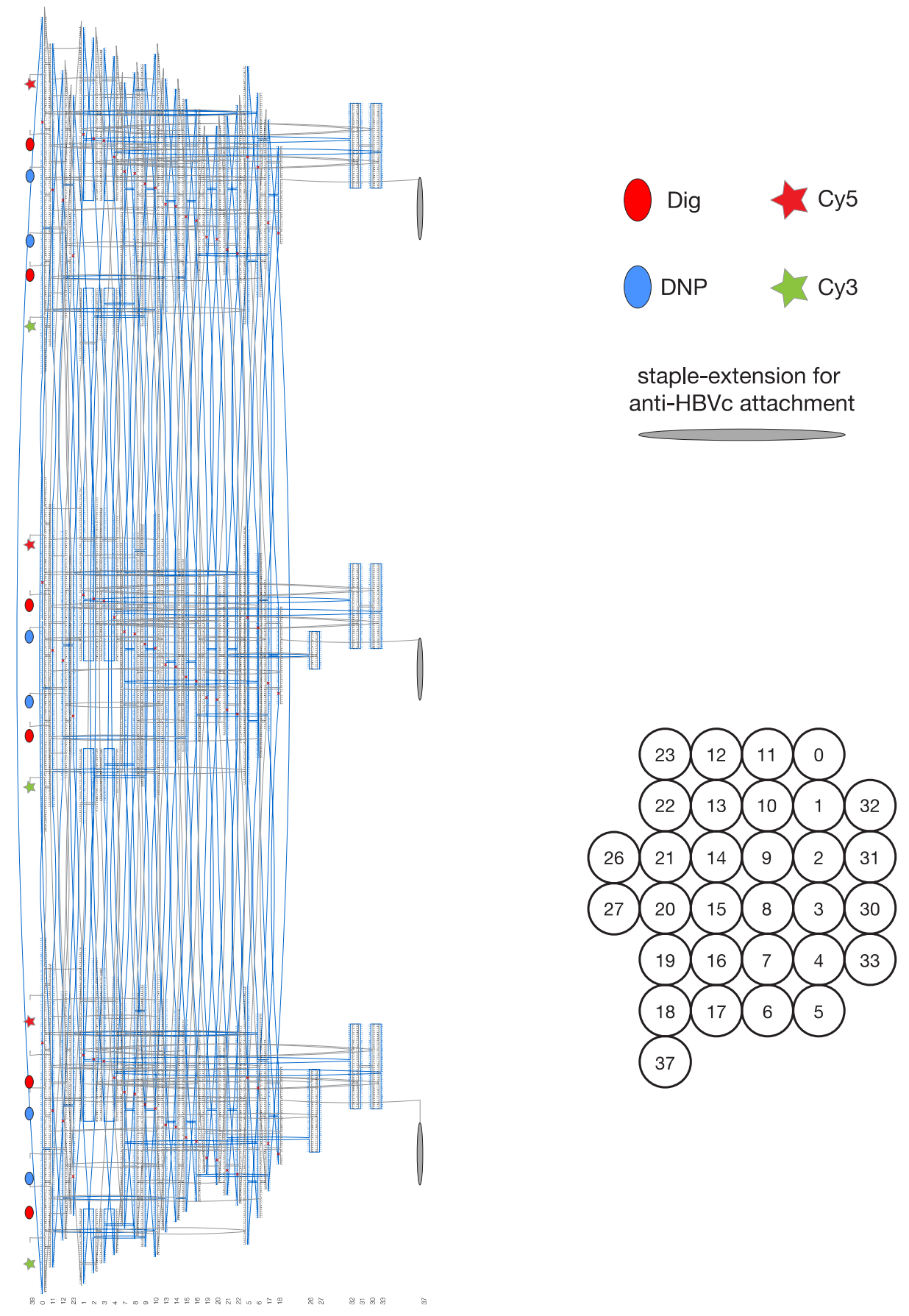

Supplementary Figure $5 \mid$ Design diagram of the $T=1$ triangle prepared with caDNAno v0.2. Oligonucleotides that connect two triangle sides have 5 additional single-stranded thymidines at the corner. The positions of the Dig, DNP, FRET, and staple-extension for the attachment of anti$\mathrm{HBV}$ c are marked in the design diagram. The helices are numbered according to the cross-section at the bottom right. 


\title{
Supplementary Note 1
}

\author{
See also Engelhardt et al. (1). For staple sequences see Supplementary Sequences.
}

\section{M13 8064:}

GGCAATGACCTGATAGCCTTTGTAGATCTCTCAAAAATAGCTACCCTCTCCGGCATTAATTTATCAGCTAGAACGGTTGAATATCATATTGATGGTGATTTGACTGTCTCCGGC CTTTCTCACCCTTTTGAATCTTTACCTACACATTACTCAGGCATTGCATTTAAAATATATGAGGGTTCTAAAAATTTTTATCCTTGCGTTGAAATAAAGGCTTCTCCCGCAAAAG TATTACAGGGTCATAATGTTTTTGGTACAACCGATTTAGCTTTATGCTCTGAGGCTTTATTGCTTAATTTTGCTAATTCTTTGCCTTGCCTGTATGATTTAATTGGATGTTAATGCT ACTACTATTAGTAGAATTGATGCCACCTTTTCAGCTCGCGCCCCAAATGAAAATATAGCTAAACAGGTTATTGACCATTTGCGAAATGTATCTAATGGTCAAACTAAATCTACT CGTTCGCAGAATTGGGAATCAACTGTTATATGGAATGAAACTTCCAGACACCGTACTTTAGTTGCATATTTAAAACATGTTGAGCTACAGCATTATATTCAGCAATTAAGCTCT AAGCCATCCGCAAAAATGACCTCTTATCAAAAGGAGCAATTAAAGGTACTCTCTAATCCTGACCTGTTGGAGTTTGCTTCCGGTCTGGTTCGCTTTGAAGCTCGAATTAAAAC GCGATATTTGAAGTCTTTCGGGCTTCCTCTTAATCTTTTTTGATGCAATCCGCTTTGCTTCTGACTATAATAGTCAGGGTAAAGACCTGATTTTTGATTTATGGTCATTCTCGTTT TCTGAACTGTTTAAAGCATTTGAGGGGGATTCAATGAATATTTATGACGATTCCGCAGTATTGGACGCTATCCAGTCTAAACATTTTACTATTACCCCCTCTGGCAAAACTTCT TTTGCAAAAGCCTCTCGCTATTTTGGTTTTTATCGTCGTCTGGTAAACGAGGGTTATGATAGTGTTGCTCTTACTATGCCTCGTAATTCCTTTTGGCGTTATGTATCTGCATTAG TTGAATGTGGTATTCCTAAATCTCAACTGATGAATCTTTCTACCTGTAATAATGTTGTTCCGTTAGTTCGTTTTATTAACGTAGATTTTTCTTCCCAACGTCCTGACTGGTATAAT GAGCCAGTTCTTAAAATCGCATAAGGTAATTCACAATGATTAAAGTTGAAATTAAACCATCTCAAGCCCAATTTACTACTCGTTCTGGTGTTTCTCGTCAGGGCAAGCCTTATT CACTGAATGAGCAGCTTTGTTACGTTGATTTGGGTAATGAATATCCGGTTCTTGTCAAGATTACTCTTGATGAAGGTCAGCCAGCCTATGCGCCTGGTCTGTACACCGTTCAT CTGTCCTCTTTCAAAGTTGGTCAGTTCGGTTCCCTTATGATTGACCGTCTGCGCCTCGTTCCGGCTAAGTAACATGGAGCAGGTCGCGGATTTCGACACAATTTATCAGGCG ATGATACAAATCTCCGTTGTACTTTGTTTCGCGCTTGGTATAATCGCTGGGGGTCAAAGATGAGTGTTTTAGTGTATTCTTTTGCCTCTTTCGTTTTAGGTTGGTGCCTTCGTA GTGGCATTACGTATTTTACCCGTTTAATGGAAACTTCCTCATGAAAAAGTCTTTAGTCCTCAAAGCCTCTGTAGCCGTTGCTACCCTCGTTCCGATGCTGTCTTTCGCTGCTGA GGGTGACGATCCCGCAAAAGCGGCCTTTAACTCCCTGCAAGCCTCAGCGACCGAATATATCGGTTATGCGTGGGCGATGGTTGTTGTCATTGTCGGCGCAACTATCGGTAT CAAGCTGTTTAAGAAATTCACCTCGAAAGCAAGCTGATAAACCGATACAATTAAAGGCTCCTTTTGGAGCCTTTTTTTTGGAGATTTTCAACGTGAAAAAATTATTATTCGCAAT TCCTTTAGTTGTTCCTTTCTATTCTCACTCCGCTGAAACTGTTGAAAGTTGTTTAGCAAAATCCCATACAGAAAATTCATTTACTAACGTCTGGAAAGACGACAAAACTTTAAGAT CGTTACGCTAACTATGAGGGCTGTCTGTGGAATGCTACAGGCGTTGTAGTTTGTACTGGTGACGAAACTCAGTGTTACGGTACATGGGTTCCTATTGGGCTTGCTATCCCTG AAAATGAGGGTGGTGGCTCTGAGGGTGGCGGTTCTGAGGGTGGCGGTTCTGAGGGTGGCGGTACTAAACCTCCTGAGTACGGTGATACACCTATTCCGGGCTATACTTATA TCAACCCTCTCGACGGCACTTATCCGCCTGGTACTGAGCAAAACCCCGCTAATCCTAATCCTTCTCTTGAGGAGTCTCAGCCTCTTAATACTTTCATGTTTCAGAATAATAGGT TCCGAAATAGGCAGGGGGCATTAACTGTTTATACGGGCACTGTTACTCAAGGCACTGACCCCGTTAAAACTTATTACCAGTACACTCCTGTATCATCAAAAGCCATGTATGAC GCTTACTGGAACGGTAAATTCAGAGACTGCGCTTTCCATTCTGGCTTTAATGAGGATTTATTTGTTTGTGAATATCAAGGCCAATCGTCTGACCTGCCTCAACCTCCTGTCAAT GCTGGCGGCGGCTCTGGTGGTGGTTCTGGTGGCGGCTCTGAGGGTGGTGGCTCTGAGGGTGGCGGTTCTGAGGGTGGCGGCTCTGAGGGAGGCGGTTCCGGTGGTGGC TCTGGTTCCGGTGATTTTGATTATGAAAAGATGGCAAACGCTAATAAGGGGGCTATGACCGAAAATGCCGATGAAAACGCGCTACAGTCTGACGCTAAAGGCAAACTTGATT CTGTCGCTACTGATTACGGTGCTGCTATCGATGGTTTCATTGGTGACGTTTCCGGCCTTGCTAATGGTAATGGTGCTACTGGTGATTTTGCTGGCTCTAATTCCCAAATGGCT CAAGTCGGTGACGGTGATAATTCACCTTTAATGAATAATTTCCGTCAATATTTACCTTCCCTCCCTCAATCGGTTGAATGTCGCCCTTTTGTCTTTGGCGCTGGTAAACCATAT GAATTTTCTATTGATTGTGACAAAATAAACTTATTCCGTGGTGTCTTTGCGTTTCTTTTAATATGTTGCCACCTTTATGTATGTATTTTCTACGTTTGCTAACATACTGCGTAATAA GGAGTCTTAATCATGCCAGTTCTTTTGGGTATTCCGTTATTATTGCGTTTCCTCGGTTTCCTTCTGGTAACTTTGTTCGGCTATCTGCTTACTTTTCTTAAAAAGGGCTTCGGTA AGATAGCTATTGCTATTTCATTGTTTCTTGCTCTTATTATTGGGCTTAACTCAATTCTTGTGGGTTATCTCTCTGATATTAGCGCTCAATTACCCTCTGACTTTGTTCAGGGTGTT CAGTTAATTCTCCCGTCTAATGCGCTTCCCTGTTTTTTATGTTATTCTCTCTGTAAAGGCTGCTATTTTCATTTTTGACGTTAAACAAAAAATCGTTTCTTATTTGGATTGGGATAA ATAATATGGCTGTTTATTTTGTAACTGGCAAATTAGGCTCTGGAAAGACGCTCGTTAGCGTTGGTAAGATTCAGGATAAAATTGTAGCTGGGTGCAAAATAGCAACTAATCTTG ATTTAAGGCTTCAAAACCTCCCGCAAGTCGGGAGGTTCGCTAAAACGCCTCGCGTTCTTAGAATACCGGATAAGCCTTCTATATCTGATTTGCTTGCTATTGGGCGCGGTAAT GATTCCTACGATGAAAATAAAAACGGCTTGCTTGTTCTCGATGAGTGCGGTACTTGGTTTAATACCCGTTCTTGGAATGATAAGGAAAGACAGCCGATTATTGATTGGTTTCTA CATGCTCGTAAATTAGGATGGGATATTATTTTTCTTGTTCAGGACTTATCTATTGTTGATAAACAGGCGCGTTCTGCATTAGCTGAACATGTTGTTTATTGTCGTCGTCTGGAC AGAATTACTTTACCTTTTGTCGGTACTTTATATTCTCTTATTACTGGCTCGAAAATGCCTCTGCCTAAATTACATGTTGGCGTTGTTAAATATGGCGATTCTCAATTAAGCCCTA CTGTTGAGCGTTGGCTTTATACTGGTAAGAATTTGTATAACGCATATGATACTAAACAGGCTTTTTCTAGTAATTATGATTCCGGTGTTTATTCTTATTTAACGCCTTATTTATCA CACGGTCGGTATTTCAAACCATTAAATTTAAGGTCAGAAGATGAAATTAACTAAAATATATTTGAAAAAGTTTTCTCGCGTTCTTTGTCTTGCGATTGGATTTGCATCAGCATTTA CATATAGTTATATAACCCAACCTAAGCCGGAGGTTAAAAAGGTAGTCTCTCAGACCTATGATTTTGATAAATTCACTATTGACTCTTCTCAGCGTCTTAATCTAAGCTATCGCTA TGTTTTCAAGGATTCTAAGGGAAAATTAATTAATAGCGACGATTTACAGAAGCAAGGTTATTCACTCACATATATTGATTTATGTACTGTTTCCATTAAAAAAGGTAATTCAAAT GAAATTGTTAAATGTAATTAATTTTGTTTTCTTGATGTTTGTTTCATCATCTTCTTTTGCTCAGGTAATTGAAATGAATAATTCGCCTCTGCGCGATTTTGTAACTTGGTATTCAAA GCAATCAGGCGAATCCGTTATTGTTTCTCCCGATGTAAAAGGTACTGTTACTGTATATTCATCTGACGTTAAACCTGAAAATCTACGCAATTTCTTTATTTCTGTTTTACGTGCA AATAATTTTGATATGGTAGGTTCTAACCCTTCCATTATTCAGAAGTATAATCCAAACAATCAGGATTATATTGATGAATTGCCATCATCTGATAATCAGGAATATGATGATAATTC CGCTCCTTCTGGTGGTTTCTTTGTTCCGCAAAATGATAATGTTACTCAAACTTTTAAAATTAATAACGTTCGGGCAAAGGATTTAATACGAGTTGTCGAATTGTTTGTAAAGTCT AATACTTCTAAATCCTCAAATGTATTATCTATTGACGGCTCTAATCTATTAGTTGTTAGTGCTCCTAAAGATATTTTAGATAACCTTCCTCAATTCCTTTCAACTGTTGATTTGCC AACTGACCAGATATTGATTGAGGGTTTGATATTTGAGGTTCAGCAAGGTGATGCTTTAGATTTTTCATTTGCTGCTGGCTCTCAGCGTGGCACTGTTGCAGGCGGTGTTAATA CTGACCGCCTCACCTCTGTTTTATCTTCTGCTGGTGGTTCGTTCGGTATTTTTAATGGCGATGTTTTAGGGCTATCAGTTCGCGCATTAAAGACTAATAGCCATTCAAAAATAT TGTCTGTGCCACGTATTCTTACGCTTTCAGGTCAGAAGGGTTCTATCTCTGTTGGCCAGAATGTCCCTTTTATTACTGGTCGTGTGACTGGTGAATCTGCCAATGTAAATAATC CATTTCAGACGATTGAGCGTCAAAATGTAGGTATTTCCATGAGCGTTTTTCCTGTTGCAATGGCTGGCGGTAATATTGTTCTGGATATTACCAGCAAGGCCGATAGTTTGAGT TCTTCTACTCAGGCAAGTGATGTTATTACTAATCAAAGAAGTATTGCTACAACGGTTAATTTGCGTGATGGACAGACTCTTTTACTCGGTGGCCTCACTGATTATAAAAACACT TCTCAGGATTCTGGCGTACCGTTCCTGTCTAAAATCCCTTTAATCGGCCTCCTGTTTAGCTCCCGCTCTGATTCTAACGAGGAAAGCACGTTATACGTGCTCGTCAAAGCAAC CATAGTACGCGCCCTGTAGCGGCGCATTAAGCGCGGCGGGTGTGGTGGTTACGCGCAGCGTGACCGCTACACTTGCCAGCGCCCTAGCGCCCGCTCCTTTCGCTTTCTTC 
CCTTCCTTTCTCGCCACGTTCGCCGGCTTTCCCCGTCAAGCTCTAAATCGGGGGCTCCCTTTAGGGTTCCGATTTAGTGCTTTACGGCACCTCGACCCCAAAAAACTTGATTT GGGTGATGGTTCACGTAGTGGGCCATCGCCCTGATAGACGGTTTTTCGCCCTTTGACGTTGGAGTCCACGTTCTTTAATAGTGGACTCTTGTTCCAAACTGGAACAACACTC AACCCTATCTCGGGCTATTCTTTTGATTTATAAGGGATTTTGCCGATTTCGGAACCACCATCAAACAGGATTTTCGCCTGCTGGGGCAAACCAGCGTGGACCGCTTGCTGCA ACTCTCTCAGGGCCAGGCGGTGAAGGGCAATCAGCTGTTGCCCGTCTCACTGGTGAAAAGAAAAACCACCCTGGCGCCCAATACGCAAACCGCCTCTCCCCGCGCGTTGG CCGATTCATTAATGCAGCTGGCACGACAGGTTTCCCGACTGGAAAGCGGGCAGTGAGCGCAACGCAATTAATGTGAGTTAGCTCACTCATTAGGCACCCCAGGCTTTACAC TTTATGCTTCCGGCTCGTATGTTGTGTGGAATTGTGAGCGGATAACAATTTCACACAGGAAACAGCTATGACCATGATTACGAATTCGAGCTCGGTACCCGGGGATCCTCAA CTGTGAGGAGGCTCACGGACGCGAAGAACAGGCACGCGTGCTGGCAGAAACCCCCGGTATGACCGTGAAAACGGCCCGCCGCATTCTGGCCGCAGCACCACAGAGTGCA CAGGCGCGCAGTGACACTGCGCTGGATCGTCTGATGCAGGGGGCACCGGCACCGCTGGCTGCAGGTAACCCGGCATCTGATGCCGTTAACGATTTGCTGAACACACCAGT GTAAGGGATGTTTATGACGAGCAAAGAAACCTTTACCCATTACCAGCCGCAGGGCAACAGTGACCCGGCTCATACCGCAACCGCGCCCGGCGGATTGAGTGCGAAAGCGC CTGCAATGACCCCGCTGATGCTGGACACCTCCAGCCGTAAGCTGGTTGCGTGGGATGGCACCACCGACGGTGCTGCCGTTGGCATTCTTGCGGTTGCTGCTGACCAGACC AGCACCACGCTGACGTTCTACAAGTCCGGCACGTTCCGTTATGAGGATGTGCTCTGGCCGGAGGCTGCCAGCGACGAGACGAAAAAACGGACCGCGTTTGCCGGAACGG CAATCAGCATCGTTTAACTTTACCCTTCATCACTAAAGGCCGCCTGTGCGGCTTTTTTTACGGGATTTTTTTATGTCGATGTACACAACCGCCCAACTGCTGGCGGCAAATGA GCAGAAATTTAAGTTTGATCCGCTGTTTCTGCGTCTCTTTTTCCGTGAGAGCTATCCCTTCACCACGGAGAAAGTCTATCTCTCACAAATTCCGGGACTGGTAAACATGGCGC TGTACGTTTCGCCGATTGTTTCCGGTGAGGTTATCCGTTCCCGTGGCGGCTCCACCTCTGAAAGCTTGGCACTGGCCGTCGTTTTACAACGTCGTGACTGGGAAAACCCTG GCGTTACCCAACTTAATCGCCTTGCAGCACATCCCCCTTTCGCCAGCTGGCGTAATAGCGAAGAGGCCCGCACCGATCGCCCTTCCCAACAGTTGCGCAGCCTGAATGGC GAATGGCGCTTTGCCTGGTTTCCGGCACCAGAAGCGGTGCCGGAAAGCTGGCTGGAGTGCGATCTTCCTGAGGCCGATACTGTCGTCGTCCCCTCAAACTGGCAGATGCA CGGTTACGATGCGCCCATCTACACCAACGTGACCTATCCCATTACGGTCAATCCGCCGTTTGTTCCCACGGAGAATCCGACGGGTTGTTACTCGCTCACATTTAATGTTGAT GAAAGCTGGCTACAGGAAGGCCAGACGCGAATTATTTTTGATGGCGTTCCTATTGGTTAAAAAATGAGCTGATTTAACAAAAATTTAATGCGAATTTTAACAAAATATTAACGT TTACAATTTAAATATTTGCTTATACAATCTTCCTGTTTTTGGGGCTTTTCTGATTATCAACCGGGGTACATATGATTGACATGCTAGTTTTACGATTACCGTTCATCGATTCTCTT GTTTGCTCCAGACTCTCA 


\title{
Supplementary Note 2
}

\author{
Oligonucleotide (staple) sequences:
}

\begin{tabular}{|c|c|}
\hline Name & Sequence \\
\hline core_1 & CCAAAAGGCACAGACAGCCCTCATTCGGCCTCAGGAAGATCGCACTC \\
\hline core_2 & ATATAAAGTATTTTTCGACAAAAGTTCATTTTTCTTCTGACCTAAA \\
\hline core_3 & AACGAGTATGTCTGGAAGTTTCATGGACGTTGACGAGGCA \\
\hline core_4 & TTACAAACTGCGGAACAAAGGGACGTTTGCCCAGTTGCAGCAAGCGGT \\
\hline core_5 & AATGCGCGAAGAATACGTGGCACAGCACTAAAGCGCGTAA \\
\hline core_6 & ATTCTGCGTTTCGCAATTTAATTGAACCAGACCCTCGTTTCCAGAGGG \\
\hline core_7 & TAGTCTTTATTAAAAAAATCGTCTTTGCAACACGCCGCTAAGAATCAG \\
\hline core_8 & GCTATTACTAAAATTCGCATTGCTTTAAACAGTT \\
\hline core_9 & GCCACCGCCACCCTCAGAGAGCCCAATAATACGAGGAAAGTGTATCATGATATAA \\
\hline core_10 & CAGTCCCGGAATTTGTTTTTGAGAGATAGACTTTACGTACAGCG \\
\hline core_11 & CTCCGTGGTGAAGGGAAAGATGATAACCTATTGTTAATGC \\
\hline core_12 & ACGTCGCGCAGAGGCGAATACAGGAGGCCGACAAATTAATACATCGGTGAATATA \\
\hline core_13 & CGGTGCGGGCCTCTTCAGCCCAATGTATAAGCAAAAGCCC \\
\hline core_14 & CTCCGGCCAGAGCACATCAGCAGCTGTTTGGAGCAATTCA \\
\hline core_15 & GGTCCGTTTTTTCGTTTTTTTCGTCGCTGGCAGCATTGCCGTTC \\
\hline core_16 & TCCTCATTAGGAGTGTGGGAGGGAGAACGGGTGGCTGTCTTTCCTTAT \\
\hline core_17 & TGCCGGACTTGATGTTTAGACTGAAAATCAGTTCATCAACGTCTGGC \\
\hline core_18 & TCAGGCTGCGCAACTTTTTTTTGGGAAGGGCGATGGCAAAGCGC \\
\hline core_19 & TGAGCAAAAGTAGCTCTCACTCCTCAAGAGAAAGTAAGCAGATA \\
\hline core_20 & GATAGAGAGTTGAGATTTAGTTTTTAATACCACATTCAACGGAACACGAGA \\
\hline core_21 & TTTGTATCATCGCCTGATGTACCGTAACAGAGGTGTTTTTATTTCTTAACGA \\
\hline core_22 & TGTTACTTAGCCGCATTAGACGGAAGACACCAAAGAACT \\
\hline core_23 & AAATATCAATAAGAAACGATTTTTTTTTTGTTTAACGTACAAAATAAGAAC \\
\hline core_24 & GCTAATGCAGAACGCGCATCAAGAAAACACAAGACTTTTTAAGAACGCGGTA \\
\hline core_25 & AGAAAAATAATCCGCGCTTAATGGGAAAAACATTAGTAA \\
\hline core_26 & GCTGCAACAAGGGAAGAAAGTTTTTGAAAGGAGCGGGCCTTGACGGTAAAG \\
\hline core_27 & GCGCGGGGAGAGGCGGGAATGCCAACGGCTGTCACTTTTTGCGCGCCTGGAG \\
\hline core_28 & TCCTCATATACCATATCAAAAATCAGTGAGGCCA \\
\hline core_29 & CCAAAAACCGTCTATCAGGG \\
\hline core_30 & TTTCATCGGCATTTTTTTTCGGACCAGAGCCTTTTTCCACCCAGAA \\
\hline core_31 & ATCATACAGGCAAGGGGAACGCCATCAGCTCATTTTTTTCGC \\
\hline core_32 & AAGACTTCAAATTTTTATCGCGTTTTAAAAAGAGGA \\
\hline core_33 & AACTTGAGATGGTTTAATTT \\
\hline core_34 & TCAGACGATCCAGCGGCCGGCCAGAATGCGGGTCGGGAAACCTGTCGAAT \\
\hline core_35 & GCTGATTGCCCTTCACCAGGGTGGAGATGATGTTATACTT \\
\hline core_36 & AGATACATAACCAAAATAGACAGCTTGATACC \\
\hline core_37 & ACTAATGCTAAAACGACGATATATTCGGTCGCAAAAGGCTGAGGCTTT \\
\hline core_38 & AATCGTAACAAACAAGGGAGAGGGTAGCTATTTTTGAGA \\
\hline core_39 & TTTTGTCAGGATATCAGGTCATTGCCTGAGAACCCAGCT \\
\hline core_40 & TTACCAGCGAATTATCACCATTACTATTCACAATTTTTCAAATAAA \\
\hline core_41 & TAAGTTTTGATGATACAAAGCCAGAATGGAAAGCGCAGT \\
\hline core_42 & СTACATTTTTTGTTCCAGTAAGCGTCATACAATCAAGTT \\
\hline core_43 & TTATTTACGAAAGCGTAACTGATAATTTAGAAGTTTTTATTAGACT \\
\hline core_44 & CACCAGAATATCATTTAATTCGACAACTCGTATTAAATC \\
\hline core_45 & AGGATTAGACATTATTAATTTTAAAAGTTTGGATTTTAA \\
\hline core_46 & GATAAGAGAAGTACGGGATTTAGTACCGTTCTATTTTTCTGATAAA \\
\hline core_47 & AATACCCAACGGAATACAAGATAGCAGCACCG \\
\hline core_48 & AGTGTACCCCGGTTGCTGCTCCAATTTTCAGGGATAGCA \\
\hline core_49 & AACGTAGAAAATTTTTACATACATAAAGGCAGTAT \\
\hline
\end{tabular}




\begin{tabular}{|c|c|}
\hline core_50 & GCTCATGGAGCACCAGCAGAAGATAAAACAGAGGTGACAAATCAACA \\
\hline core_51 & GCCTTGCTGGTTTTTTAATATCCAGAACAAACTCAA \\
\hline core_52 & CAGTTCCTGATTATCTTTTTCTTCGTGGTGCTGGTCTGG \\
\hline core_53 & AACTCCAAAGGTGTTTAGCTATATTTTCATTTGGGGCGTTGTACCAA \\
\hline core_54 & AGCCCGAGGAAGATTAGGAACCCATAAATTGTGTCGAAAAAGGGAAC \\
\hline core_55 & ACTATCGATCCTGATAACCGCAATTTGCGTATTGGGCGCCGCCTGGC \\
\hline core_56 & CCAGTAGCACCGTCACCGACTTGAAACGCTAAATAACATA \\
\hline core_57 & САCTAAAACATTTTTCTCATCTTTGGACATTTTTCAACCATCGCCCA \\
\hline core_58 & CGCTCACTGCTTTTTCGCTTTCCACGGGTTTTTCGTTTTCACGGTC \\
\hline core_59 & GCCGAACAAAGTTACCAGTAAGACTCAGAGAGATACTGAACA \\
\hline core_60 & TTAATGCCAGAATCGAGAATATAAACGGTGTACCAACTTTGAAAGAGG \\
\hline core_61 & CCGAGTAAAAGAGTCTGTCTTGCCTGTCCTCGTTCAGGGCGC \\
\hline core_62 & TCATAGCCCCCTTATCGGATAAGGCGGGGTTTTGCTCACCA \\
\hline core_63 & GAGAGCCAGCAGCTTTTTAATGTATCTGGTCTTTTTGTTGGGGCGG \\
\hline core_64 & AGCATTAACATCCTTTTTATAACATAAAGCTTTTTTAATCGGCGAG \\
\hline core_65 & AATAAAGCAAATTGTAAACGCGGCCACCCTC \\
\hline core_66 & GCCTTTAGCGTCACAAAGGATAAAAATTTTTAGAACCCT \\
\hline core_67 & TCAAGTTTGGCATGATAAGGAAACAGAGCAAGTTTAGTACACGTTGT \\
\hline core_68 & ATCAAAATACATGAAAGTATATATCATTTCA \\
\hline core_69 & AACACCGCCTGCAATCAGCCGCCACCAG \\
\hline core_70 & TCAGTATTTAACATCACCATCACGTTAAAGGGGATTCGCCGTTGTGT \\
\hline core_71 & CAATCAAAAAAATCTAAAGCATAGATTTTCAAACAGAAATAAAGACGTGGGAA \\
\hline core_72 & CAAACCCTCTGAATAATGGAGACAACGTCAG \\
\hline core_73 & GTGGCATCAATTCACCTCAAATATATATCTTT \\
\hline core_74 & CTGAAAAGAAAGCGGAATAAATCAGATAGCGTGAGTAACAAGTTTGA \\
\hline core_75 & TGCAACTAGTCATTTTTGCGGATGGTTTTTTTAGAGCTTAATTGCT \\
\hline core_76 & ATGACCCAATCACCATCAATATGATTTTTTTTCATTGACCATTAGATACA \\
\hline core_77 & ATTCTGGCCAACTTTTTGAGATAGAACCCTATTTAGAG \\
\hline core_78 & AGCCGCCGCAGGTCAGACGATTGGCTTTTTTTTGACATTAGCAAGGCCGGA \\
\hline core_79 & TCTGACCTATTGGCAGATTCACCAGTTTTTCACACGACCAGTAATA \\
\hline core_80 & GGAATTGGTCAATAGATAATACATTTTTTTTGAGGGCCCTAAAACATCGCC \\
\hline core_81 & AGGCCGGATGTCAATCATATTTAAACGAGGCACAA \\
\hline core_82 & CAATGAAACCATCAAGGGTGAGAAGATCTACAAAGGCATTAGAGCCAG \\
\hline core_83 & TTGACAGGCAGTGCCTTGAGTACCCATCCTATTTT \\
\hline core_84 & TACCGAACGAACCCGCCGCCAGCACTCTGAATTTACCTGAATGGCTAT \\
\hline core_85 & CTAATAGAATTATCATCATAGTCGGGCAACAGAAC \\
\hline core_86 & ATGGTCAATAACCAGCACTAACAACTTTGCCCGAACGGTTGATTCCCA \\
\hline core_87 & CAGCCAGCAAACTACAACTTTTTCCTGTAGCATTCAGCCTTTAATTGTAT \\
\hline core_88 & CCACGGGATTTCATTTGATTTTTTTACCTTTTTTAATAACTATATGTAAAT \\
\hline core_89 & GCGGCCTTCCCACGCAACTTTTTAGCTTACGGCTGTGCCGGTGCCCCCTGC \\
\hline core_90 & AGGTAAATATTGTTTTTTCGGAAATTATTCATGCCAGTT \\
\hline core_91 & CGCCACCCTCAGAACCGGAATAGCGCAATAATAGGACTGTAGCGCGT \\
\hline core_92 & CCATGTTTACATAGCTATCTTACAGGAAACAATG \\
\hline core_93 & TTAAAGGTGCCAAAGACAAAAGGGCTTTTTACATTCAACCGATTGA \\
\hline core_94 & TGATTGCTTTGAATAGTACCTTTCCGTTGTAGCCACAGTGCCACGCT \\
\hline core_95 & CATTCGCCATGTCATAAATATTCAGCCCAATACT \\
\hline core_96 & CGGCAAACGCGTACGCCAGAATCACGATTTTAGA \\
\hline core_97 & ACCCGTCGGATTCTCAGCCAGCTGTCTTTACTGATACTAATAGTAGT \\
\hline core_98 & ATAATTCGCATTAAATGTGATTGAATCCCTTTTTCTCAGGAAACCA \\
\hline core_99 & GAGAGGGTCCGTACTCAGGCGAAGCCCTTTTTTTTTAATCGGCGAA \\
\hline core_100 & ACGTCAGAGAGAAACAATACTGAGAAGTGTTTTTTTTTTCGATGCTG \\
\hline core_101 & AACCACCACCAGGCCACCA \\
\hline core_102 & TTTAAATGCAATGCCTGAGTAATGTGTCGGGAGA \\
\hline core_103 & CATATATTAATCAGTAGCGAGGAACCGCCT \\
\hline core_104 & TGCTGTAGCTCATTTTTCATGTTTTAAATAACGAACTA \\
\hline core_105 & AGCCTTTATTTCAACGCAAATTAAGCTAAAGATT \\
\hline core_106 & СССTCAGTTTTCATACCCTCAGAAAATAACAGTGCCCGCCTGAACAATTACC \\
\hline
\end{tabular}




$$
\begin{aligned}
& \text { core_107 } \\
& \text { core_108 } \\
& \text { core_109 } \\
& \text { core_110 } \\
& \text { core_111 } \\
& \text { core_112 } \\
& \text { core_113 } \\
& \text { core_114 } \\
& \text { core_115 } \\
& \text { core_116 } \\
& \text { core_117 } \\
& \text { core_118 } \\
& \text { core_119 } \\
& \text { core_120 } \\
& \text { core_121 } \\
& \text { core_122 } \\
& \text { core_123 } \\
& \text { core_124 } \\
& \text { core_125 } \\
& \text { core_126 } \\
& \text { core_127 } \\
& \text { core_128 } \\
& \text { core_129 } \\
& \text { core_130 } \\
& \text { core_131 } \\
& \text { core_132 } \\
& \text { core_133 } \\
& \text { core_134 } \\
& \text { core_135 } \\
& \text { core_136 }
\end{aligned}
$$

protrusion_recess_side1_1 protrusion_recess_side1_2 protrusion_recess_side1_3 protrusion_recess_side1_4 protrusion_recess_side1_5 protrusion_recess_side1_6 protrusion_recess_side1_7 protrusion_recess_side1_8 protrusion_recess_side1_9 protrusion_recess_side1_10 protrusion_recess_side1_11 protrusion_recess_side1_12 protrusion_recess_side1_13 protrusion_recess_side1_14 protrusion_recess_side2_1 protrusion_recess_side2_2 protrusion_recess_side2_3 protrusion_recess_side2_4 protrusion_recess_side2_5 protrusion_recess_side2_6 protrusion_recess_side2_7 protrusion_recess_side2_8 protrusion_recess_side2_9 protrusion_recess_side2_10 protrusion_recess_side2_11 protrusion_recess_side2_12 protrusion_recess_side2_13
TTATTTATCCCCCAATAGCAAGCAAATCAGATTTTTTTTAGAAGGCTTATCCG CGAACGTGGCGAGATAGGGTTGAGTGTTGTTCTTTTTAGTTTGGAACAAGAG TCCTTGCGGGAGGTTTTGAA AGGTAGAAAGATCAACGTAACAAAGCTGCTCATTTTTTCAGTGAATAAGGCT ATACCGGGCACATTAATTGCGTTGTCCACTATGGAAAGCCGG GCCAGCGGGAGGTGTCCAGCATCAATCCCGTAAAAAAAGCCGCACAG CTGATGCAAATCCAATGTTATTTTAGTTAATGTAAAGTAATTCTGTCTTA TGGGTTATATGGAAACAGTACATACCAAGCTTTCAGAGGTGGAGCCG CGCATAACAAGAGGCAAAAGAATATGCCCTGAACATTATTAC GGTTTATCAGCTTGCTCGGTTGCGCCGACAATACCCCCAGCGATTATATTA GTTAGCATATTTCGGGAAACAAACCTGTTTATCAACAATAAACCAAT CAAAATCAAACGTCACAGAAAATTGAAACGCAGAGAATTAAACCCACA GCAGACGGTCAATCATTCCGCGACATAATCAGAAATATTTCTCAGAG CAAAAATGCATTTTCGTTTGAAATACCGACCGGCTTAGGTATTGAGCG ATTTACGAGCATGTAGAGATAAGTTATAAACAATTCTGAACACCGGA GCTAGGGCAGCTAACTGGTTTCTGCCAGCACGTACCTGCACACGTATA GCTGGCAAGTTGCTTTGACTGCACTCTGTGGT TTCACCAGTGACACTATCATAACCGGAAGCATCAGAAGC AAAATAGCAAAGTCAGAGGAGAAAACTTTTTC TTTAATGGAGCCAGTAATAAGAGAGTATTCTAAACAGCCATA CAGAAAACGAGAATGACCTTGCATCAAAGTTTTGACCAGACG TTTTTTAGTTGCTATTTTGCACCTTCATCAAGAGTAGCGCATAGGCTGGCTGGT GGGGGTGAACCATCACCCAAGAGAACAAGCAAGCCGAAGTACCGCACTCATCTG TGGCGTGAATTACCTTATGCGGTGGTTCCGAAATCGGAAAATCCTGTTTGATAG AAAACGACGGCCAGTGAATCAATAAAATAGCACTAATATC GGGGGATGAATAAATTTTTGTTAAATCAAAA ACATCGACATAAAAAAGCGGGGTCCAGGAACGACGTGCTT GCAGAAACGAAAGGATTAGGATTATGCCGTC GGGGACGACGACAGTAAGTTAGCGGCGGAATCTGCAAAAG CGTAATGGATATTATTTGCACGTAAGGTTTA ATAGCTTAGATTAGAAGAGTCCAGTCACG GCTATTAATTAATAACCTCCGTGTGATAACCCTGAACAGCCTTTA AGAGACTATGACTGAAGACGATAACCTTGCTTCTGTAAATCGTC AGATCTTGACAAGAACCGGATATTCACCAAGCGCGAA TAATTACTAGAAATCAAGAATCCTGAATCTTACCGCCATTTGCAATCAAT CCATATTTAACAAGTAATTTACTCCCGATTTCCAGAGCCTAATT TATGTGAGCCTTTTTTTTCCCTTGTAGGGCTTAATTGAGAATCG CACCCTCAGAAGCAGTTGGGTAACGCCAGGGTTTTCCAATAGTGAATT GTTTAGTAGTTAAATAGCTCAACAAGAATCCTTGAAAACATAGCG TATCAAAATCATAGGTCTGAGAATTGAGTTACCA GTATAAAGCCAACAGAATAAACACCGGAATCA CGTCACCAGTACTTTCCGGCACCGCTTCTGGTGCCTGCTGCAAGGCGATTATGGCGAAA ACAAAGTACAATTCCTGAGTTT ATAAGGCTCAACATCGCCATATGCGTTATACAAATTCTTACCA GAAGCATAAAGTGGGGTGCCTAAGGGCGCTAAAGGGAGCCCCCG GAATTCGTCGTCCGTGGCTCACAAACTGTTGCCCTGCGGCTGGTA AGTTACAAAAGAAATTTCTGCTCATTTGCCGCCAGCACATCCCTTACA TACATTTAACAAACGGATAACCTCACCGGAAACAAAGCGGATCAAACTTAAAAAGAGAC TTCTTCGAATCCTGTAAAGCATGGTCATAGCTGTTTCCTGTGT AATAAACAACATCGAAATTAAT CTGGTGTGTTCAGCAAATCAGCGGGAGCTAATATCTTCTTTG CCTTTTTATTTTCATCGTAGGAATCACAGACGACGAC ATGGGTAAAGGTTGTCATAAAGTTGGGCG GTTAACGGCGCGCTCTCTTTTTTCGCACTCAATCCGCCGGGCGC ATCCCCGGGTACCCACTACTCGAGGTGCCGTAAAGACAATATTGACGCTC GGTTGCGGTATGATGCCGGGTCGTGCCTGGTACTATGGTGTAGCG GAAATTGTTATCCAGCCTCCTCACAGTTGAGG 


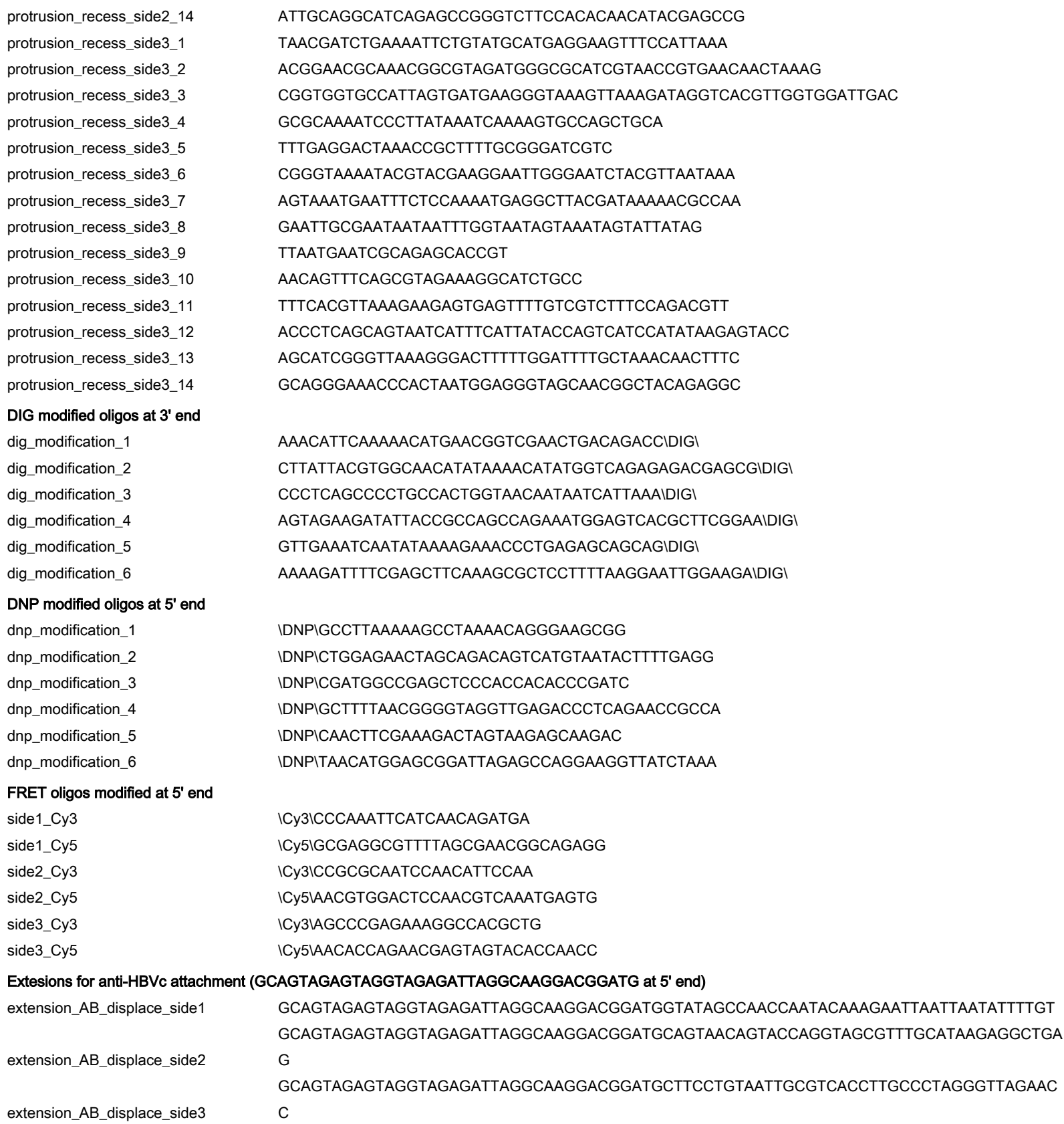

1. F. A. S. Engelhardt et al., Custom-Size, Functional, and Durable DNA Origami with Design-Specific Scaffolds. ACS Nano 13, 5015-5027 (2019). 